\title{
Dynamics Analysis of a Mathematical Model for New Product Innovation Diffusion
}

\author{
Chunru Li $\mathbb{D D}^{1}$ and Zujun Ma $\mathbb{D}^{2}$ \\ ${ }^{1}$ Jiangsu Vocational College of Electronics and Information, Huaian 223003, China \\ ${ }^{2}$ School of Economics and Management, Southwest Jiaotong University, Chengdu 610031, China \\ Correspondence should be addressed to Chunru Li; crli1976@126.com
}

Received 16 June 2020; Revised 11 August 2020; Accepted 30 October 2020; Published 20 November 2020

Academic Editor: Youssef N. Raffoul

Copyright (c) 2020 Chunru Li and Zujun Ma. This is an open access article distributed under the Creative Commons Attribution License, which permits unrestricted use, distribution, and reproduction in any medium, provided the original work is properly cited.

\begin{abstract}
In this paper, a mathematical model with time-delay-related parameters and media coverage to describe the diffusion process of new products is proposed, in which the time-delay-related parameters denote the stage in which potential customers decide whether to adopt a new product. Then, the stability and the Hopf bifurcation of the proposed model are analyzed in detail. The center manifold theorem and the normal form theory are used to investigate the stability of the bifurcating periodic solution. Moreover, a numerical simulation is conducted to investigate the difference between the model with delay-dependent parameters and that with delay-independent parameters. The results show that there is significant difference between the two models.
\end{abstract}

\section{Introduction}

The diffusion of a product innovation has traditionally been defined as a process of communication among members of a social system overtime in a certain way, consisting of innovation, communication channels, time, and social systems. With the high-pace growth of the launch of new products, decision makers need to pay much attention to how to push these new products into market successfully. Fourt and Woodlock [1] proposed the first purchase model to describe the diffusion process, which is the earliest and quite popular. Bass [2] tried to establish mathematical models to describe the penetration and saturation of the diffusion process of a new product. The Bass model is the main driving force of the diffusion research and is expressed as follows:

$$
\frac{\mathrm{d} N(t)}{\mathrm{d} t}=p[m-N(t)]+\frac{q}{m} N[m-N(t)],
$$

where $N(t)$ is the total number of adopters of a product for time $t, m$ stands for the total number of potential future customers, and $p$ and $q$ denote coefficients of innovation and imitation, respectively. The first term in the model represents the adoption by innovators, while the latter represents the adoption by imitators.
In the past several years, a number of modifications to the Bass model have been proposed and studied, and the Bass model has become more generalized [3-10]. In [6], Kalish investigated a method for maximizing the firm profit cash flow and proposed the optimal advertising strategy, which has issues in innovation. Robinson and Lakhani [7] also considered the impact of the product price in the proposed model. Other variable models were incorporated by Kalish and Lilien [11] in their study of advertising strategies. We refer readers to [12-15] for some other related works.

Note that none of the abovementioned models considered the factor of delay. In fact, for a new product, a person usually takes some time to consider accepting or rejecting it. For this reason, Fanelli and Maddalena [16] proposed a model with time-delay parameters to describe the diffusion process of a new product:

$$
\begin{aligned}
\frac{\mathrm{d} A(t)}{\mathrm{d} t}= & {[h+\alpha A(t-\tau)][C-A(t-\tau)] e^{-\rho \tau}-\delta A(t) } \\
& -\gamma A(t) A(t-\tau),
\end{aligned}
$$

where $A(t)$ is the total population of adopters at time $t, C$ stands for the total number of potential adopters, and $h=e^{\eta(i-c)}$, in which $i>0$ can be used as a governing 
incentive, $c>0$ is the cost of production, and $\eta$ is a positive constant. $\alpha>0$ denotes the valid communication between the adopters and potential adopters, $\delta>0$ is used to describe the dismissal rate of the adopters of a product, and $\gamma>0$ stands for the rate of valid contact between the adopters at time $t$ and those at time $t-\tau . k_{\tau}=e^{-\rho \tau}$, where $\rho>0$ denotes the percentage of potential individuals who do not adopt the product after they have evaluated it. The stability of the equilibrium point was also researched by the authors. Then, Ballestra et al. [17] studied stability switches and Hopf bifurcations of the model (2).

On the contrary, with the rapid development of communication technology, as the representative of new media, microblogs and WeChat have become the new means of information dissemination. New e-commerce has come under a high degree of concern, with the proliferation of innovative products increasing more and more with new media for marketing communication. The main difference between new media and traditional media is the carrier of communication, which determines the method of information dissemination and whether the user will accept the information in a different state. New media use digital technology, wireless communication network satellite channels, and mobile terminals, to provide users with the dissemination of information and service patterns. Regarding new media as a third channel for the dissemination of product information, the classic model is not considered when assessing what role new media plays in the product information dissemination process and how much of its impact is worth exploring.

Joydip Dhar et al. [18] proposed the following model to examine the effect of a media report on the spreading of a product:

$$
\left\{\begin{array}{l}
\frac{\mathrm{d} N}{\mathrm{~d} t}=r-d_{1} N-\left(\beta_{1}+\frac{\beta_{2} A}{m+A}\right) N A+\delta R, \\
\frac{\mathrm{d} A}{\mathrm{~d} t}=\left(\beta_{1}+\frac{\beta_{2} A}{m+A}\right) N A-\left(d_{1}+\nu\right) A, \\
\frac{\mathrm{d} R}{\mathrm{~d} t}=\nu A-\left(d_{1}+\delta\right) R,
\end{array}\right.
$$

where $N(t), A(t)$, and $R(t)$ denote the nonadopter class, adopter class, and frustrated class, respectively. $\beta_{1}$ is the contact rate before the media alert, and $\beta(A)=\beta_{1}+\left(\beta_{2} A / m+A\right)$ is the contact rate after the media alert. Their results show that the media has a great effect on the dynamics of the model.

Therefore, in this paper, based on the works of $[16,18]$, a model with time-delay parameters and the media effect is proposed to investigate the impacts of the media on innovation diffusion.

The remainder of this paper is presented as follows. In Section 2, we have developed and analyzed a model to incorporate the media impact considering two classes of population, namely, potential adopter and adopter. In Section 3, the local stability is investigated, and the existence of Hopf bifurcation is studied. In Section 4, a formula is established and used to determine the direction and stability of bifurcation. Finally, numerical simulations are provided to verify the theoretical predictions in the analysis presented in Sections 3 and 4.

\section{Mathematical Model}

This section describes a delayed mathematical model for new product innovation diffusion. Our goal is to create a realistic model that can provide wide insights into the diffusion of a product innovation.

Generally, a social system consists of many people who may not adopt a new product. We divide these people into two classes depending on their different states: one is the potential adopter class, and the other is the adopter class, denoted by $P(t)$ and $A(t)$, respectively, at time $t$. To model the impact of the new product innovation diffusion on a social system, the following assumptions are imposed:

(i) In a social system, not everyone knows about the product; hence, only those who know the product information can become potential adopters. Therefore, we consider that the recruitment rate of the population that will join the nonadopter class is a constant $r$.

(ii) In a social system, when the potential adopters make contact with the adopters, they usually need some time to consider accepting or rejecting the new product; that is, there exists a delay $\tau$. $\rho$ denotes the percentage of persons who decide not to adopt the technology after they have evaluated it, and $e^{-\rho \tau}$ denotes the individuals who remain interested but make no decision.

(iii) We assume that the adopters at time $t$ are also affected by the adopters at time $t-\tau$. Let $\alpha$ denote the rate of valid contacts between the adopters at time $t$ and those at time $t-\tau$.

(iv) We assume that the acceptance of new products by people is affected by media reports. Here, we use $\beta_{1}$ and $\beta(A)=\beta_{1}+\left(\beta_{2} A / m+A\right)$ to represent the contact rate before and after media reports, respectively. The function $\left(\beta_{2} A / m+A\right)$ is adopted to model the media reports and is used to describe the transmission rate when adopters appear and are reported. Obviously, when $A \longrightarrow \infty$, the function $\left(\beta_{2} A / m+A\right)$ approaches the maximum value $\beta_{2}$, and when the reported adopter arrives at $m$, the function $\left(\beta_{2} A / m+A\right)$ equals to half the maximum $\beta_{2}$.

(v) Both classes have a death rate $\delta$, which is proportional to the existing population. Indeed, if a potential user is not interested in the product, or a person who has adopted the product, after a period of time, he will never use the product.

Therefore, the model is governed by the following system of equations: 


$$
\left(\begin{array}{c}
\frac{\mathrm{d} P}{\mathrm{~d} t}=r+\alpha A A(t-\tau)-\left(\beta_{1}+\frac{\beta_{2} A}{m+A}\right) P A e^{-\rho \tau}-\delta P, \\
\frac{\mathrm{d} A}{\mathrm{~d} t}=\left(\beta_{1}+\frac{\beta_{2} A}{m+A}\right) P A e^{-\rho \tau}-\alpha A A(t-\tau)-\delta A,
\end{array}\right)
$$

where $r, \alpha, \beta_{1}, \beta_{2}$, and $\delta$ are all positive constants. Summarising, the meaning of the parameters is shown in Table 1.

In the following, we study the stability and Hopf bifurcation for system (4) with delay $\tau$ as the bifurcation parameter.

\section{Stability and Hopf Bifurcation}

In the following, we consider the stability and Hopf bifurcation of the equilibria of system (4). First, we find all possible equilibria of system (4). According to system (4), the equilibria should satisfy

$$
\left(\begin{array}{c}
r+\alpha A^{2}-\left(\beta_{1}+\frac{\beta_{2} A}{m+A}\right) P A e^{-\rho \tau}-\delta P=0 \\
\left(\beta_{1}+\frac{\beta_{2} A}{m+A}\right) P A e^{-\rho \tau}-\alpha A^{2}-\delta A=0 .
\end{array}\right)
$$

Obviously, $E_{0}=(r / \delta, 0)$ is an equilibrium of system (4). For other equilibria, adding the two equations of (5) yields

$$
A=\frac{r-\delta P}{\delta} .
$$
obtain

Substituting equation (6) into the first equation of (5), we

$$
B_{1}(\tau) P^{3}+B_{2}(\tau) P^{2}+B_{3}(\tau) P+B_{4}(\tau)=0,
$$

where

$$
\begin{aligned}
B_{1}(\tau)= & \delta^{3} \delta+\beta_{1} \delta^{2} e^{-\rho \tau}+\beta_{2} \delta^{3} e^{-\rho \tau}, \\
B_{2}(\tau)= & -3 \delta^{2} \alpha r-\delta^{3} \alpha m-2 \beta_{1} \delta^{2} r e^{-\rho \tau}-2 \beta_{2} \delta^{2} r e^{-\rho \tau} \\
& -\beta_{1} \delta^{3} m e^{-\rho \tau}, \\
B_{3}(\tau)= & \delta^{3} r+3 \delta \alpha r^{2}+\beta_{1} \delta e^{-\rho \tau} r^{2}+\beta_{2} \delta e^{-\rho \tau} r^{2} \\
& +2 \delta^{2} \alpha m r+\beta_{1} \delta^{2} e^{-\rho \tau} m r, \\
B_{4}(\tau)= & -m d_{2}^{3} r-\delta^{2} r^{2}-\alpha m \delta r^{2}-\alpha r^{3} .
\end{aligned}
$$

Obviously, $B_{1}(\tau)>0$ and $B_{4}(\tau)<0$. Therefore, equation (7) has at least a positive solution $P^{*}$. If $r-\delta P^{*}>0$, then system (4) has at least a positive equilibrium $E^{*}=\left(P^{*}, A^{*}\right)$.

In the following, we consider the stability of the equilibria of system (4) by analyzing the corresponding characteristic equations. First, we assume the following:

$$
\left(H_{1}\right) \delta^{2}>\left(\beta_{1}+\beta_{2}\right) r e^{-\rho \tau} .
$$

Theorem 1. If $\left(H_{1}\right)$ holds, then the equilibrium $E_{0}$ is globally asymptotically stable.
Proof. For $E_{0}$, the characteristic equation becomes

$$
(\lambda+\delta)\left(\lambda-\frac{r}{\delta} \beta_{1} e^{-\rho \tau}+\delta\right)=0 .
$$

Obviously, $\quad \lambda_{1}=-\delta<0 \quad$ and $\quad \lambda_{2}=(r / \delta) \beta_{1} e^{-\rho \tau}-\delta$. Therefore, if $\delta^{2}>\beta_{1} r e^{-\rho \tau}$, then $\lambda_{2}<0$. Thus, the equilibrium $E_{0}$ is locally asymptotically stable.

Adding the two equations in model (4), we have

$$
\frac{\mathrm{d}(P+A)}{\mathrm{d} t}=r-\delta(P+A) .
$$

Therefore, one obtained that $P+A \leq(r / \delta)$, which implies that $P \leq(r / \delta)-A$.

On the contrary, from the second equation of system (4), we obtain

$$
\begin{aligned}
\frac{\mathrm{d} A}{\mathrm{~d} t} & =\left(\beta_{1}+\frac{\beta_{2} A}{m+A}\right) P A e^{-\rho \tau}-\alpha A A(t-\tau)-\delta A \\
& \leq\left(\beta_{1}+\beta_{2}\right)\left(\frac{r}{\delta}-A\right) A e^{-\rho \tau}-\alpha A A(t-\tau)-\delta A \\
& \leq A\left(\left(\beta_{1}+\beta_{2}\right) \frac{r}{\delta} e^{-\rho \tau}-\left(\beta_{1}+\beta_{2}\right) A e^{-\rho \tau}-\alpha A(t-\tau)-\delta\right) .
\end{aligned}
$$

Because $\left(H_{1}\right)$ holds, by comparison principle, we have $A(t) \longrightarrow 0(t \longrightarrow \infty)$. Therefore, for an arbitrary $\varepsilon>0$, there exists $t_{1}(>\tau)$ such that, for any $t>t_{1}, A(t)<\varepsilon$.

From the first equation of system (4), there exists a $t_{2}>t_{1}$ such that

$$
\begin{aligned}
\frac{\mathrm{d} P}{\mathrm{~d} t}= & r+\alpha A A(t-\tau)-\left(\beta_{1}+\frac{\beta_{2} A}{m+A}\right) P A e^{-\rho \tau} \\
& -\delta P \leq r+\varepsilon^{2}-\delta P .
\end{aligned}
$$

Again by the comparison principle, we have $P(t) \longrightarrow(r / \delta)(t \longrightarrow \infty)$. Based on the above discussions, we find that if $\left(H_{1}\right)$ holds, then the boundary equilibrium is globally asymptotically stable.

Remark 1. Obviously, if $\tau \longrightarrow \infty$, then the term $e^{-\rho \tau} \longrightarrow 0$. Hence, the condition $\left(H_{1}\right)$ must hold so that the solution of system (4) converges to equilibrium $E_{0}$. This means that when the period of evaluation of new product adoption becomes very long, the number of adopters decreases since they no longer adopt the product.

Now, we discuss the stability of the positive equilibrium $E^{*}$. The linearization of (4) at a constant solution $E^{*}$ can be expressed by

$$
\left(\begin{array}{c}
\frac{\mathrm{d} P}{\mathrm{~d} t}=\gamma_{1} P+\left(-\beta_{2} P^{*} e^{-\rho \tau} \gamma_{2}+\gamma_{3}\right) A+\alpha A^{*} A(t-\tau), \\
\frac{\mathrm{d} P}{\mathrm{~d} t}=\gamma_{4} P+\beta_{2} P^{*} e^{-\rho \tau} \gamma_{2} A-\alpha A^{*} A(t-\tau),
\end{array}\right)
$$

where 
TABLE 1: Meaning of the parameters.

\begin{tabular}{lr}
\hline Parameters & Meaning \\
\hline$r$ & Recruitment rate of the population that will join the nonadopter class \\
$\tau$ & Potential adopters need some time to consider accepting or rejecting the new product \\
$\alpha$ & Rate of valid contacts between the adopters at time $t$ and those at time $t-\tau$ \\
$\beta_{1}, \beta_{2}$ & Contact rate \\
$m$ & Half saturation coefficient \\
$\delta$ & Death rate \\
$\rho$ & Percentage of persons who decide not to adopt the technology after they have evaluated it
\end{tabular}

$$
\begin{aligned}
& \gamma_{1}=-\frac{r+\alpha A^{*^{2}}}{P^{*}}, \\
& \gamma_{2}=\frac{A m}{\left(m+A^{*}\right)^{2}}, \\
& \gamma_{3}=\frac{\delta P^{*}-r}{A^{*}}, \\
& \gamma_{4}=\frac{\alpha A^{*^{2}}+d_{2} A^{*}}{P^{*}} .
\end{aligned}
$$

The characteristic equation associated with system (14) is

$$
R(\lambda, \tau)+Q(\lambda, \tau) e^{-\lambda \tau}=0
$$

where

$$
\begin{aligned}
& R(\lambda, \tau)=\lambda^{2}+a(\tau) \lambda+c(\tau) \\
& Q(\lambda, \tau)=b(\tau) \lambda+d(\tau)
\end{aligned}
$$

and $a(\tau), b(\tau), c(\tau)$, and $d(\tau)$ are defined as follows:

$$
\begin{aligned}
& a(\tau)=-\gamma_{1}-\beta_{2} P^{*} e^{-\rho \tau} \gamma_{2}, \\
& b(\tau)=\alpha A^{*}, \\
& c(\tau)=\beta_{2} P^{*} e^{-\rho \tau} \gamma_{2}\left(\gamma_{4}+\gamma_{1}\right)-\gamma_{4} \gamma_{3}, \\
& d(\tau)=-\alpha A^{*}\left(\gamma_{1}+\gamma_{4}\right) .
\end{aligned}
$$

When $\tau=0$, equation (16) becomes

$$
\lambda^{2}+(a(0)+b(0)) \lambda+c(0)+d(0)=0 .
$$

We make the following assumptions:

$$
\begin{aligned}
\left(H_{2}\right) \beta_{2} P^{*} \gamma_{2}< & \alpha A^{*}, d_{2} A^{*}>r, \\
& \cdot\left(H_{3}\right) \delta\left(r+A^{*} \beta_{2} P^{*} e^{-\rho \tau} \gamma_{2}+\alpha A^{* 2}\right) \\
& -\delta \alpha A^{*} P^{*}-r \beta_{2} P^{*} e^{-\rho \tau} \gamma_{2}<0 .
\end{aligned}
$$

Lemma 1. If $\left(\mathrm{H}_{2}\right)$ holds, then the positive equilibrium of system (4) is locally asymptotically stable with $\tau=0$.

Proof. Let $\lambda_{1}$ and $\lambda_{2}$ be two roots of equation (19). If $\left(H_{2}\right)$ holds, then we have

$$
\begin{aligned}
\lambda_{1}+\lambda_{2} & =-(a(0)+b(0))=\gamma_{1}-\alpha A^{*}+\beta_{2} P^{*} \gamma_{2}<0, \\
\lambda_{1} \lambda_{2} & =c(0)+d(0)=\left(\gamma_{4}+\gamma_{1}\right)\left(\beta_{2} P^{*} \gamma_{2}-\alpha A^{*}\right)-\gamma_{4} \gamma_{3}>0 .
\end{aligned}
$$

This means that all the roots of equation (19) have negative real parts. Thus, equilibrium $E^{*}$ of system (4) with $\tau=0$ is locally asymptotically stable.

Remark 2. (H2) implies that only when $A^{*}$ is larger than a threshold value, the positive equilibrium of system (4) is locally asymptotic stable with $\tau=0$.

If $\tau>0$, then $R(\lambda, \tau)$ and $Q(\lambda, \tau)$ are delay dependent. Based on the above analysis, a necessary condition for the local stability switch of $E^{*}$ is that equation (16) has purely imaginary solutions. Assume that $i \omega(\omega>0)$ is a root of equation (16). Then, $\omega$ should satisfy the following equation:

$$
-\omega^{2}+i a(\tau) \omega+c(\tau)+(i b(\tau) \omega+d(\tau))(\cos (\omega \tau)-i \sin (\omega \tau))=0,
$$

which implies that

$$
\left(\begin{array}{c}
\omega^{2}-c(\tau)=d(\tau) \cos (\omega \tau)+b(\tau) \omega \sin (\omega \tau), \\
a(\tau) \omega=-b(\tau)(\tau) \omega \cos (\omega \tau)+d(\tau) \sin (\omega \tau) .
\end{array}\right)
$$

It follows that

$$
\left(\begin{array}{l}
\sin (\omega \tau)=\frac{\left(\omega^{2}-c(\tau)\right) b(\tau) \omega+a(\tau) d(\tau) \omega}{\omega^{2} b^{2}(\tau)+d^{2}(\tau)} \\
\cos (\omega \tau)=\frac{\left(\omega^{2}-c(\tau)\right) d(\tau)-\omega^{2} a(\tau) b(\tau)}{\omega^{2} b^{2}(\tau)+d^{2}(\tau)}
\end{array}\right)
$$

As we know, $\cos ^{2}(\omega \tau)+\sin ^{2}(\omega \tau)=1$; thus, we obtain

$$
\omega^{4}+\left(a^{2}(\tau)-2 c(\tau)-b^{2}(\tau)\right) \omega^{2}+c^{2}(\tau)-d^{2}(\tau)=0 .
$$

where $a(\tau), b(\tau), c(\tau)$, and $d(\tau)$ are as defined in (18). Obviously, we have the following Lemma.

Lemma 2. If $\left(\mathrm{H}_{2}\right)$ and $\left(\mathrm{H}_{3}\right)$ hold, then equation (25) has a unique root.

According to equation (25), (26) has a unique root denoted by $\omega_{0}$, where

$$
\begin{aligned}
\omega_{0}^{2} & =\frac{1}{2}\left(\left(b^{2}(\tau)+2 c(\tau)-a^{2}(\tau)\right)+\Delta^{1 / 2}\right), \\
\Delta & =\left(b^{2}(\tau)+2 c(\tau)-a^{2}(\tau)\right)^{2}-4\left(c^{2}(\tau)-d^{2}(\tau)\right) .
\end{aligned}
$$

By equation (23), we have 


$$
\cos \left(\omega_{0} \tau\right)=\frac{\left(w_{0}^{2}-c(\tau)\right) d(\tau)-a(\tau) b(\tau) w_{0}^{2}}{b^{2}(\tau) w_{0}^{2}+d^{2}(\tau)} .
$$

Thus, if we denote

$$
\begin{array}{r}
\tau_{j}(\tau)=\frac{1}{\omega_{0}}\left(\arccos \left(\frac{\left(w_{0}^{2}-c(\tau)\right) d(\tau)-a(\tau) b(\tau) w_{0}^{2}}{b^{2}(\tau) w_{0}^{2}+d^{2}(\tau)}\right)+2 j \pi\right) \\
j=0,1,2, \ldots,
\end{array}
$$

then $\pm i \omega_{0}$ is a pair of purely imaginary roots of (16) with $\tau=\tau_{j}(\tau)$. In addition, the stability switches take place at the zeros of the functions:

$$
S_{j}(\tau):=\tau-\tau_{j}(\tau), \quad \text { for some } j \in N_{0} .
$$

Recently, Beretta and Kuang [19] studied the stability switches of some delay differential systems with delay-dependent parameters and established a geometrical criterion that reveals the existence of purely imaginary roots for a characteristic equation with delay-dependent coefficients. Therefore, according to [19], one has the following results.

Lemma 3. The characteristic equation (16) admits a pair of simple conjugate pure imaginary roots $\lambda= \pm \omega_{0}\left(\tau_{j}\right)$ and $\left(\omega_{0}\left(\tau_{j}\right)>0\right)$ if $S_{j}(\tau)=0$ for some $j \in N_{0}$. If $S_{j}(\tau)=0$, this pair of simple conjugate pure imaginary roots crosses the imaginary axis from left to right (as $\tau$ increases) if $\delta_{j}(\tau)>0$ and from right to left if $\delta_{j}(\tau)<0$. The crossing direction of the pair of simple conjugate pure imaginary roots through the imaginary axis is determined by

$$
\delta\left(\tau_{j}\right):=\operatorname{sign}\left\{\left.\frac{\mathrm{dRe} \lambda}{d \tau}\right|_{\lambda=i \omega_{0}\left(\tau_{j}\right)}\right\}=-\operatorname{sign}\left\{\left.\frac{\mathrm{d} S_{j}(\tau)}{\mathrm{d} \tau}\right|_{\tau=\tau_{j}}\right\} .
$$

Based on the above discussions, we have the following results.

Theorem 2. If $\left(\mathrm{H}_{2}\right)$ and $\left(\mathrm{H}_{3}\right)$ hold, assume further that $S_{j}(\tau)=0$ and $\delta\left(\tau_{j}\right)>0$. Then, when $\tau=\tau_{j}$, the Hopf bifurcation occurs. That is, system (4) has a branch of periodic solutions bifurcating from $E^{*}$ near $\tau=\tau_{j}$.

Remark 3. If the function $S_{j}(\tau)=0$ has two or more roots, then a stability switch may occur in system (4).

\section{Stability and Direction of the Hopf Bifurcation}

In this section, we investigate the direction and stability of period solutions bifurcating from the positive equilibrium $E^{*}$ by applying the center manifold theorem and normal form theory developed in [20].

Denote $\tau_{j}$ by $\tau^{*}$ and introduce the new parameter $\mu=\tau-\tau^{*}$. Then, normalize the delay $\tau$ by the time-scaling $t \longrightarrow t / \tau$. Thus, system (4) can be rewritten as

$$
\frac{\mathrm{d} U(t)}{\mathrm{d} t}=L\left(\tau^{*}\right)\left(U_{t}\right)+F\left(U_{t}, \mu\right)
$$

where

$$
\begin{aligned}
L(\mu)(\varphi) & =\mu\left(\begin{array}{c}
\gamma_{1} \varphi_{1}(0)+\left(\gamma_{3}-\beta_{2} P^{*} e^{-\rho \tau^{*}} \gamma_{2}\right) \varphi_{2}(0)+\alpha \varphi_{2}(-1) \\
\gamma_{4} \varphi_{1}(0)+\beta_{2} P^{*} e^{-\rho \tau^{*}} \gamma_{2} \varphi_{2}(0)-\alpha \varphi_{2}(-1)
\end{array}\right), \\
F(\varphi, \mu) & =L(\mu) \varphi+f(\varphi, \mu), \\
f(\varphi, \mu) & =\left(\tau^{*}+\mu\right)\left(\begin{array}{l}
-e^{-\rho \tau^{*}}\left(\beta_{1}+\beta_{2} \gamma 5\right) \varphi_{1}(0) \varphi_{2}(0)-\beta_{2} P^{*} e^{-\rho \tau^{*}} \gamma_{6} \varphi_{2}^{2}(0)+\alpha \varphi_{2}(0) \varphi_{2}(-1) \\
e^{-\rho \tau^{*}}\left(\beta_{1}+\beta_{2} \gamma 5\right) \varphi_{1}(0) \varphi_{2}(0)+\beta_{2} P^{*} e^{-\rho \tau^{*}} \gamma_{6} \varphi_{2}^{2}(0)-\alpha \varphi_{2}(0) \varphi_{2}(-1)
\end{array}\right)+\text { h.o.t. } \\
\gamma_{5} & =\frac{2 A(m+A)-A^{2}}{m+A}, \\
\gamma_{6} & =\frac{2 m^{2}}{(m+A)^{3}},
\end{aligned}
$$

for $\varphi=\left(\varphi_{1}, \varphi_{2}\right)^{T} \in \mathscr{C}$.

Then, the linearized equation of $(32)$ at the origin $(0,0)$ is

$$
\frac{\mathrm{d} U(t)}{\mathrm{d} t}=L\left(\tau^{*}\right)\left(U_{t}\right)
$$
$\mathscr{C}:$

Let $\mathscr{C}:=C\left([-1,0], \mathbb{R}^{2}\right)$. Consider the following FDE on

$$
\dot{z}=L\left(\tau^{*}\right)\left(z_{t}\right)
$$

where $L\left(\tau^{*}\right)$ is a continuous linear function mapping $C\left([-1,0], \mathbb{R}^{2}\right)$ to $\mathbb{R}^{2}$. By the Riesz representation theorem, there is a $2 \times 2$ matrix function $\eta(\theta, \tau) \quad(-1 \leq \theta \leq 0)$ whose elements are of bounded variation such that

$$
L\left(\tau^{*}\right)(\varphi)=\int_{-1}^{0}\left[\mathrm{~d} \eta\left(\theta, \tau^{*}\right)\right] \varphi(\theta), \quad \text { for } \varphi \in C .
$$


Actually, we can choose

$$
\begin{aligned}
\eta\left(\theta, \tau^{*}\right)= & \tau^{*}\left(\begin{array}{cc}
\gamma_{1} & \gamma_{3}-\beta_{2} P^{*} e^{-\rho \tau^{*}} \gamma_{2} \\
\gamma_{4} & \beta_{2} P^{*} e^{-\rho \tau^{*}} \gamma_{2}
\end{array}\right) \delta(\theta) \\
& -\tau^{*}\left(\begin{array}{cc}
0 & \alpha \\
0 & -\alpha
\end{array}\right) \delta(\theta+1)
\end{aligned}
$$

where $\delta$ is the Dirac delta function.

Let $A\left(\tau^{*}\right)$ represent the infinitesimal generator of the semigroup induced by the solutions of (35) and $A^{*}$ be the formal adjoint of $A\left(\tau^{*}\right)$ under the bilinear pairing

$$
\begin{aligned}
(\psi, \phi) & =(\psi(0), \phi(0))-\int_{-1}^{0} \int_{\xi=0}^{\theta} \psi(\xi-\theta) \mathrm{d} \eta(\theta) \phi(\xi) \mathrm{d} \xi \\
& =(\psi(0), \phi(0))+\tau^{*} \int_{-1}^{0} \psi(\theta+1)\left(\begin{array}{cc}
0 & \alpha \\
0 & -\alpha
\end{array}\right) \phi(\theta) \mathrm{d} \theta
\end{aligned}
$$

for $\phi \in C, \psi \in C^{*}=C\left([0,1], R^{2}\right)$. Then, $A\left(\tau^{*}\right)$ and $A^{*}$ are a pair of adjoint operators. It is easily obtained that $A\left(\tau^{*}\right)$ has a pair of simple purely imaginary eigenvalues $\pm i \omega_{0} \tau^{*} . A\left(\tau^{*}\right)$ and $A^{*}$ are a pair of adjoint operators, so they are also eigenvalues of $A^{*}$.

Let $P$ and $P^{*}$ be the center spaces. Then, $P^{*}$ is the adjoint space of $P$ and $\operatorname{dim} P=\operatorname{dim} P^{*}=2$.

Lemma 4. Let

$$
\left\{\begin{array}{l}
\sigma=\frac{i \omega_{0}-\gamma_{1}}{\gamma_{3}-\beta_{2} P^{*} \gamma_{2} e^{-\rho \tau}+\alpha e^{-\omega_{0} \tau}} \\
\sigma^{*}=\frac{i \omega-\gamma_{1}}{\omega_{4}}
\end{array}\right.
$$

Then, for $-1 \leq \theta \leq 0$,

$$
\begin{aligned}
& r_{1}(\theta)=(1, \sigma)^{T} e^{i \omega_{0} \tau^{*} \theta}, \\
& r_{2}(\theta)=\bar{r}_{1}(\theta),
\end{aligned}
$$

are a basis of $P$ associated with $\Lambda_{0}$, and for $0 \leq s \leq 1$,

$$
\begin{aligned}
& q_{1}(s)=e^{-i \omega_{0} \tau^{*} s}\left(1, \sigma^{*}\right), \\
& q_{2}(s)=\bar{q}_{1}(s),
\end{aligned}
$$

are a basis of $Q$ associated with $\Lambda_{0}$.

Let $\Phi=\left(\Phi_{1}, \Phi_{2}\right)$ and $\Psi^{*}=\left(\Psi_{1}^{*}, \Psi_{2}^{*}\right)^{T}$ with

$$
\begin{aligned}
\Phi_{1}(\theta) & =\frac{r_{1}(\theta)+r_{2}(\theta)}{2}=\left(\begin{array}{c}
\operatorname{Re}\left\{e^{i \omega_{0} \tau^{*} \theta}\right\} \\
\operatorname{Re}\left\{\sigma e^{i \omega_{0} \tau^{*} \theta}\right\}
\end{array}\right) \\
& =\left(\begin{array}{c}
\cos \omega_{0} \tau^{*} \theta \\
\frac{L_{1} \cos \left(\omega_{0} \tau^{*} \theta\right)+L_{2} \omega \sin \left(\omega_{0} \tau^{*} \theta\right)}{h_{1}^{2}+\alpha^{2}}
\end{array}\right),
\end{aligned}
$$

$$
\begin{aligned}
h_{1} & =\gamma_{3}-\beta_{2} P^{*} \gamma_{2} e^{-\rho \tau}+\alpha \cos (\omega \tau), \\
L_{1} & =-h_{1} \gamma_{1}-\alpha \omega \sin (\omega \tau), \\
L_{2} & =-h_{1} \omega+\alpha \gamma_{1} \sin (\omega \tau), \\
\Phi_{2}(\theta) & =\frac{r_{1}(\theta)-r_{2}(\theta)}{2 i}=\left(\begin{array}{c}
\operatorname{Im}\left\{e^{i \omega_{0} \tau^{*} \theta}\right\} \\
\operatorname{Im}\left\{\sigma^{*} e^{i \omega_{0} \tau^{*} \theta}\right\}
\end{array}\right) \\
& =\left(\begin{array}{c}
\sin \omega_{0} \tau^{*} \theta \\
\frac{L_{3} \cos \left(\omega_{0} \tau^{*} \theta\right)+L_{4} \sin \left(\omega_{0} \tau^{*} \theta\right)}{h_{1}^{2}+\alpha^{2}}
\end{array}\right),
\end{aligned}
$$

where

$$
\begin{aligned}
& L_{3}=h_{1} \omega-\alpha \gamma_{1} \sin (\omega \tau), \\
& L_{4}=-h_{1} \gamma_{1}-\omega \sin (\omega \tau),
\end{aligned}
$$

for $\theta \in[-1,0]$ and

$$
\begin{aligned}
\Psi_{1}^{*}(s) & =\frac{q_{1}(s)+q_{2}(s)}{2}=\left(\begin{array}{c}
\operatorname{Re}\left\{e^{-i \omega_{0} \tau^{*} s}\right\} \\
\operatorname{Re}\left\{\sigma^{*} e^{-i \omega_{0} \tau^{*} s}\right\}
\end{array}\right) \\
& =\left(\begin{array}{c}
\cos \omega_{0} \tau^{*} s \\
\left.-\frac{\omega \sin \left(\omega \tau^{*} s\right)-\gamma_{1} \cos \left(\omega \tau^{*} s\right)}{\gamma_{4}}\right) \\
\Psi_{2}^{*}(s)
\end{array}=\frac{q_{1}(s)-q_{2}(s)}{2 i}=\left(\begin{array}{c}
\operatorname{Im}\left\{e^{-i \omega_{0} \tau^{*} s}\right\} \\
\operatorname{Im}\left\{\sigma^{*} e^{-i \omega_{0} \tau^{*} s}\right\}
\end{array}\right)\right. \\
& =\left(\begin{array}{c}
\sin \omega_{0} \tau^{*} s \\
\frac{\gamma_{1} \sin \left(\omega_{0} \tau^{*} s\right)+\omega_{0} \cos \left(\omega_{0} \tau^{*} s\right)}{\gamma_{4}}
\end{array}\right),
\end{aligned}
$$

for $s \in[0,1]$. According to (38), we get $\left(\Psi_{1}^{*}, \Phi_{1}\right)$ and $\left(\Psi_{1}^{*}, \Phi_{2}\right)$. It is noted that

$$
\begin{aligned}
& \left(q_{1}, r_{1}\right)=\left(\Psi_{1}^{*}, \Phi_{1}\right)-\left(\Psi_{2}^{*}, \Phi_{2}\right)+i\left[\left(\Psi_{1}^{*}, \Phi_{2}\right)+\left(\Psi_{2}^{*}, \Phi_{1}\right)\right], \\
& \left(q_{1}, r_{1}\right)=1+\sigma \sigma^{*}+\sigma \tau^{*} \alpha\left(1-\sigma^{*}\right) e^{-i \omega_{0} \tau^{*}}:=W^{*} .
\end{aligned}
$$

Consequently, we obtain

$$
\begin{aligned}
& \left(\Psi_{1}^{*}, \Phi_{1}\right)-\left(\Psi_{2}^{*}, \Phi_{2}\right)=\operatorname{Re}\left\{W^{*}\right\} \\
& \left(\Psi_{1}^{*}, \Phi_{2}\right)+\left(\Psi_{2}^{*}, \Phi_{1}\right)=\operatorname{Im}\left\{W^{*}\right\} .
\end{aligned}
$$

Let $\left(\Psi^{*}, \Phi\right)=\left(\Psi_{j}^{*}, \Phi_{k}\right)(j, k=1,2)$. Clearly,

$$
\Psi=\left(\Psi_{1}, \Psi_{2}\right)^{T}=\left(\Psi^{*}, \Phi\right)^{-1} \Psi^{*}
$$

is a new basis $\psi$ of $Q$.

Furthermore, we define $f_{0}=\left(\xi_{0}^{1}, \xi_{0}^{2}\right)$, where

$$
\begin{aligned}
& \xi_{0}^{1}=\left(\begin{array}{l}
1 \\
0
\end{array}\right), \\
& \xi_{0}^{2}=\left(\begin{array}{l}
0 \\
1
\end{array}\right) .
\end{aligned}
$$

where 
Let $c \cdot f_{0}$ be defined by

$$
c \cdot f_{0}=c_{1} \xi_{0}^{1}+c_{2} \xi_{0}^{2},
$$

for $c=\left(c_{1}, c_{2}\right)^{T}, c_{j} \in R(j=1,2)$.

Then, the center space of equation (34) is given by $P_{\mathrm{CN}} \mathscr{C}$, where

$$
P_{\mathrm{CN}} \varphi=\Phi\left(\Psi,\left\langle\varphi, f_{0}\right\rangle\right) \cdot f_{0}, \quad \varphi \in c,
$$

and $\mathscr{C}=P_{\mathrm{CN}} \mathscr{C} \oplus P_{S} \mathscr{C}$, where $P_{S} \mathscr{C}$ represents the complementary subspace of $P_{\mathrm{CN}} \mathscr{C}$.

Assume $A_{\tau^{*}}$ is defined as follows:

$$
A_{\tau^{*}} \varphi(\theta)=\dot{\varphi}(\theta)+X_{0}(\theta)\left[L\left(\tau^{*}\right)(\varphi(\theta))-\dot{\varphi}(0)\right], \quad \varphi \in B \mathscr{C},
$$

where $X_{0}:[-1,0] \longrightarrow B(X, X)$ is given by

$$
X_{0}(\theta)= \begin{cases}0, & -1 \leq \theta<0 \\ I, & \theta=0\end{cases}
$$

Therefore, the solutions of (32) and (34) induce the infinitesimal generator $A_{\tau^{*}}$, and it can be described by the following equation:

$$
\dot{U}_{t}=A_{\tau^{*}} U_{t}+X_{0} F\left(U_{t}, \mu\right) .
$$

By $\mathscr{C}=P_{\mathrm{CN}} \mathscr{C} \oplus P_{S} \mathscr{C}$ and (51), the solution of (32) can be described as

$$
U_{t}=\Phi\left(x_{1}(t), x_{2}(t)\right)^{T} \cdot f_{0}+h\left(x_{1}, x_{2}, \mu\right),
$$

where

$$
\begin{aligned}
\left(x_{1}(t), x_{2}(t)\right)^{T} & =\left(\Psi,\left\langle U_{t}, f_{0}\right\rangle\right), \\
h\left(x_{1}, x_{2}, \mu\right) & \in P_{s} c,
\end{aligned}
$$

and $h(0,0,0)=D h(0,0,0)=0$. Particularly, on the center manifold, the solution of (32) is as follows:

$$
U_{t}^{*}=h\left(x_{1}, x_{2}, 0\right)+\Phi\left(x_{1}(t), x_{2}(t)\right)^{T} \cdot f_{0} .
$$

Let $z=x_{1}-i x_{2}$. By $r_{1}=\Phi_{1}+i \Phi_{2}$, then (57) is rewritten as

$$
U_{t}^{*}=W(z, \bar{z})+\frac{1}{2}\left(r_{1} z+\overline{r_{1}} \bar{z}\right) \cdot f_{0},
$$

where $W(z, \bar{z})=h(z+\bar{z} / 2,-z-\bar{z} / 2 i, 0)$. In addition, $z$ satisfies the following equation:

$$
\begin{aligned}
\dot{z} & =i \omega_{0} \tau^{*} z+g(z, \bar{z}), \\
g(z, \bar{z}) & =\left(\Psi_{1}(0)-i \Psi_{2}(0)\right)\left\langle F\left(U_{t}^{*}, 0\right), f_{0}\right\rangle .
\end{aligned}
$$

Denote

$$
\begin{gathered}
W(z, \bar{z})=W_{20} \frac{z^{2}}{2}+W_{11} z \bar{z}+W_{02} \frac{\bar{z}^{2}}{2}+\cdots, \\
g(z, \bar{z})=g_{20} \frac{z^{2}}{2}+g_{11} z \bar{z}+g_{02} \frac{\bar{z}^{2}}{2}+\cdots
\end{gathered}
$$

By (58), we get

$$
\begin{aligned}
\left\langle F\left(U_{t}^{*}, 0\right), f_{0}\right\rangle= & \frac{\tau^{*} z^{2}}{4}\left(\begin{array}{l}
-e^{-\rho \tau^{*}}\left(\beta_{1}+\beta_{2} \gamma_{5}\right)-\beta_{2} P^{*} e^{-\rho \tau^{*}} \gamma_{6} \sigma^{2}+\alpha \sigma^{2} e^{-i \omega \tau^{*}} \\
e^{-\rho \tau^{*}}\left(\beta_{1}+\beta_{2} \gamma_{5}\right)+\beta_{2} P^{*} e^{-\rho \tau^{*}} \gamma_{6} \sigma^{2}-\alpha \sigma^{2} e^{-i \omega \tau^{*}}
\end{array}\right) \\
& +\frac{\tau^{*} z \bar{z}}{4}\left(\begin{array}{c}
-e^{-\rho \tau^{*}}\left(\beta_{1}+\beta_{2} \gamma_{5}\right)(\sigma+\bar{\sigma})-2 \beta_{2} P^{*} e^{-\rho \tau^{*}} \gamma_{6} \sigma \bar{\sigma}+\alpha \sigma \bar{\sigma}\left(e^{-i \omega \tau^{*}}+e^{i \omega \tau^{*}}\right) \\
e^{-\rho \tau^{*}}\left(\beta_{1}+\beta_{2} \gamma_{5}\right)(\sigma+\bar{\sigma})+2 \beta_{2} P^{*} e^{-\rho \tau^{*}} \gamma_{6} \sigma \bar{\sigma}-\alpha \sigma \bar{\sigma}\left(e^{-i \omega \tau^{*}}+e^{i \omega \tau^{*}}\right)
\end{array}\right) \\
& +\frac{\tau^{*} \bar{z}^{2}}{4}\left(\begin{array}{c}
-e^{-\rho \tau^{*}}\left(\beta_{1}+\beta_{2} \gamma_{5}\right) \bar{\sigma}-\beta_{2} P^{*} e^{-\rho \tau^{*}} \gamma_{6} \bar{\sigma}^{2}+\alpha \bar{\sigma}^{2} e^{i \omega \tau^{*}} \\
e^{-\rho \tau^{*}}\left(\beta_{1}+\beta_{2} \gamma_{5}\right) \bar{\sigma}+\beta_{2} P^{*} e^{-\rho \tau^{*}} \gamma_{6} \bar{\sigma}^{2}-\alpha \bar{\sigma}^{2} e^{i \omega \tau^{*}}
\end{array}\right) \\
& +\frac{\tau^{*}}{4}\left(\begin{array}{c}
+e^{-\rho \tau^{*}}\left(\beta_{1}+\beta_{2} \gamma_{5}\right)\left(2 w_{11}^{(2)}(0)+w_{20}^{(2)}(0)+2 \sigma w_{11}^{(1)}(0)+\bar{\sigma} w_{20}^{(1)}(0)-\beta_{2} P^{*} e^{-\rho \tau^{*}} \gamma_{6}(2 \bar{\sigma}) w_{20}^{(2)}(0)\right) \\
\left\langle 4 \sigma w_{11}^{(2)}(0)+\alpha\left(2 \sigma w_{11}^{(2)}(-1)+\bar{\sigma} w_{20}^{(2)}(-1)+2 \sigma w_{11}^{(2)}(0) e^{-i \omega \tau^{*}}+\bar{\sigma} e^{i \omega \tau^{*}} w_{20}^{(1)}(0)\right), 1\right\rangle \\
\left\langle\beta_{1}+\beta_{2} \gamma_{5}\right)\left(2 w_{11}^{(2)}(0)+w_{20}^{(2)}(0)+2 \sigma w_{11}^{(1)}(0)+\bar{\sigma} w_{20}^{(1)}(0)+\beta_{2} P^{*} e^{-\rho \tau^{*}} \gamma_{6}(2 \bar{\sigma}) w_{20}^{(2)}(0)\right. \\
\left.+4 \sigma w_{11}^{(2)}(0)-\alpha\left(2 \sigma w_{11}^{(2)}(-1)+\bar{\sigma} w_{20}^{(2)}(-1)+2 \sigma w_{11}^{(2)}(0) e^{-i \omega \tau^{*}}+\bar{\sigma} e^{i \omega \tau^{*}} w_{20}^{(1)}(0)\right), 1\right\rangle
\end{array}\right) z^{2} \bar{z}+\cdots \\
\left\langle W_{i j}^{n}(\theta), 1>=\right. & \frac{1}{\pi} \int_{0}^{\pi} W_{i j}^{n}(\theta)(x) \mathrm{d} x .
\end{aligned}
$$


Let $\left(\psi_{1}, \psi_{2}, \psi_{3}\right)=\Psi_{1}(0)-i \Psi_{2}(0)$. Thus, by (60)-(62), we can obtain the following quantities:

$$
\begin{aligned}
g_{20}= & \frac{\tau^{*}}{2}\left[\left(-e^{-\rho \tau^{*}}\left(\beta_{1}+\beta_{2} \gamma_{5}\right)-\beta_{2} P^{*} e^{-\rho \tau^{*}} \gamma_{6} \sigma^{2}+\alpha \sigma^{2} e^{-i \omega \tau^{*}}\right) \psi_{1}+\left(e^{-\rho \tau^{*}}\left(\beta_{1}+\beta_{2} \gamma_{5}\right)+\beta_{2} P^{*} e^{-\rho \tau^{*}} \gamma_{6} \sigma^{2}-\alpha \sigma^{2} e^{-i \omega \tau^{*}}\right) \psi_{2}\right], \\
g_{11}= & \frac{\tau^{*}}{4}\left[\left(-e^{-\rho \tau^{*}}\left(\beta_{1}+\beta_{2} \gamma_{5}\right)(\sigma+\bar{\sigma})-2 \beta_{2} P^{*} e^{-\rho \tau^{*}} \gamma_{6} \sigma \bar{\sigma}+\alpha \sigma \bar{\sigma}\left(e^{-i \omega \tau^{*}}+e^{i \omega \tau^{*}}\right)\right) \psi_{1}\right. \\
& +\left(e^{-\rho \tau^{*}}\left(\beta_{1}+\beta_{2} \gamma_{5}\right)(\sigma+\bar{\sigma})+2 \beta_{2} P^{*} e^{-\rho \tau^{*}} \gamma_{6} \sigma \bar{\sigma}-\alpha \sigma \bar{\sigma}\left(e^{-i \omega \tau^{*}}+e^{i \omega \tau^{*}}\right) e^{-\rho \tau^{*}}\left(\beta_{1}+\beta_{2} \gamma_{5}\right)(\sigma+\bar{\sigma})\right. \\
& +2 \beta_{2} P^{*} e^{-\rho \tau^{*}} \gamma_{6} \sigma \bar{\sigma}-\alpha \sigma \bar{\sigma}\left(e^{-i \omega \tau^{*}}+e^{i \omega \tau^{*}}\right) \psi_{2}, \\
g_{02}= & \frac{\tau^{*}}{2}\left[\left(-e^{-\rho \tau^{*}}\left(\beta_{1}+\beta_{2} \gamma_{5}\right) \bar{\sigma}-\beta_{2} P^{*} e^{-\rho \tau^{*}} \gamma_{6} \bar{\sigma}^{2}+\alpha \bar{\sigma}^{2} e^{i \omega \tau^{*}}\right) \overline{\psi_{1}}+\left(e^{-\rho \tau^{*}}\left(\beta_{1}+\beta_{2} \gamma_{5}\right) \bar{\sigma}+\beta_{2} P^{*} e^{-\rho \tau^{*}} \gamma_{6} \bar{\sigma}^{2}-\alpha \bar{\sigma}^{2} e^{i \omega \tau^{*}}\right) \overline{\psi_{2}}\right], \\
g_{21}= & \left\langle-e^{-\rho \tau^{*}}\left(\beta_{1}+\beta_{2} \gamma_{5}\right)\left(2 w_{11}^{(2)}(0)+w_{20}^{(2)}(0)+2 \sigma w_{11}^{(1)}(0)+\bar{\sigma} w_{20}^{(1)}(0)-\beta_{2} P^{*} e^{-\rho \tau^{*}} \gamma_{6}\left(2 \bar{\sigma} w_{20}^{(2)}(0)+4 \sigma w_{11}^{(2)}(0)\right)\right.\right. \\
& \left.+\alpha\left(2 \sigma w_{11}^{(2)}(-1)+\bar{\sigma} w_{20}^{(2)}(-1)+2 \sigma w_{11}^{(2)}(0) e^{-i \omega \tau^{*}}+\bar{\sigma} e^{i \omega \tau^{*}} w_{20}^{(1)}(0)\right), 1\right\rangle \psi_{1} \\
& \left.+4 \sigma w_{11}^{(2)}(0)-\alpha\left(2 \sigma w_{11}^{(2)}(-1)+\bar{\sigma} w_{20}^{(2)}(-1)+2 \sigma w_{11}^{(2)}(0) e^{-i \omega \tau^{*}}+\bar{\sigma} e^{i \omega \tau^{*}} w_{20}^{(1)}(0)\right), 1\right\rangle \psi_{2} . \\
& +\left\langlee ^ { - \rho \tau ^ { * } } ( \beta _ { 1 } + \beta _ { 2 } \gamma _ { 5 } ) \left( 2 w_{11}^{(2)}(0)+w_{20}^{(2)}(0)+2 \sigma w_{11}^{(1)}(0)+\bar{\sigma} w_{20}^{(1)}(0)+\beta_{2} P^{*} e^{-\rho \tau^{*}} \gamma_{6}(2 \bar{\sigma}) w_{20}^{(2)}(0)\right.\right.
\end{aligned}
$$

Notice that $g_{21}$ concludes $W_{20}(\theta), W_{11}(\theta)$ for $\theta \in[-1,0]$. From $(61)$, we can easily obtain that

$$
\begin{gathered}
\dot{W}(z, \bar{z})=W_{20} z \dot{z}+W_{11}(\dot{z} \bar{z}+z \bar{z})+W_{02} \bar{z} \bar{z}+\cdots, \\
A_{\tau^{*}} W=A_{\tau^{*}} W_{20} \frac{z^{2}}{2}+A_{\tau^{*}} W_{11} z \bar{z}+A_{\tau^{*}} W_{02} \frac{\bar{z}^{2}}{2}+\cdots .
\end{gathered}
$$

Furthermore, by [20], $W(z(t), \bar{z}(t))$ satisfies

$$
\dot{W}=A_{\tau^{*}} W+H(z, \bar{z})
$$

where

$$
H(z, \bar{z})=H_{20} \frac{z^{2}}{2}+H_{11} z \bar{z}+H_{02} \frac{\bar{z}^{2}}{2}+\cdots,
$$

with $H_{i j} \in P_{S} \mathscr{C}, i+j=2$. Thus, from (58) and (65)-(67), we can obtain that

$$
\left\{\begin{array}{l}
\left(2 i \omega_{0} \tau^{*}-A_{\tau^{*}}\right) W_{20}=H_{20}, \\
-A_{\tau^{*}} W_{11}=H_{11}
\end{array}\right.
$$

It is noted that $A_{\tau^{*}}$ has two pure imaginary roots $\pm i \omega_{0} \tau^{*}$. Thus, (67) has a unique solution $W_{i j}(i+j=2)$ in $P_{S} \mathscr{C}$ given by

$$
\left\{\begin{array}{l}
W_{20}=\left(2 i \omega_{0} \tau^{*}-A_{\tau^{*}}\right)^{-1} H_{20}, \\
W_{11}=-A_{\tau^{*}}^{-1} H_{11}
\end{array}\right.
$$

From (68), we obtain that

$$
H(z, \bar{z})=-\Phi(\theta) \Psi(0)<F\left(U_{t}^{*}, 0\right), \quad f_{0}>\cdot f_{0}
$$

$$
=-\frac{1}{4}\left[g_{20} r_{1}(\theta)+\bar{g}_{02} r_{2}(\theta)\right] z^{2} \cdot f_{0}
$$

$$
-\frac{1}{2}\left[g_{11} r_{1}(\theta)+\bar{g}_{11} r_{2}(\theta)\right] z \bar{z} \cdot f_{0}+\cdots,
$$

for $-1 \leq \theta<0$.

Therefore, for $-1 \leq \theta<0$, 


$$
\begin{aligned}
& H_{20}(\theta)=-\frac{1}{2}\left[g_{20} r_{1}(\theta)+\bar{g}_{02} r_{2}(\theta)\right] \cdot f_{0} \\
& H_{11}(\theta)=-\frac{1}{2}\left[g_{11} r_{1}(\theta)+\bar{g}_{11} r_{2}(\theta)\right] \cdot f_{0} \\
& H(z, \bar{z})(0)=F\left(U_{t}^{*}, 0\right)-\Phi\left(\Psi,\left\langle F\left(U_{t}^{*}, 0\right), f_{0}\right\rangle\right) \cdot f_{0} \\
& H_{20}(0)=\frac{\tau^{*}}{2}\left(\begin{array}{c}
-e^{-\rho \tau^{*}}\left(\beta_{1}+\beta_{2} \gamma_{5}\right)-\beta_{2} P^{*} e^{-\rho \tau^{*}} \gamma_{6} \sigma^{2}+\alpha \sigma^{2} e^{-i \omega \tau^{*}} \\
e^{-\rho \tau^{*}}\left(\beta_{1}+\beta_{2} \gamma_{5}\right)+\beta_{2} P^{*} e^{-\rho \tau^{*}} \gamma_{6} \sigma^{2}-\alpha \sigma^{2} e^{-i \omega \tau^{*}}
\end{array}\right) \\
& -\frac{1}{2}\left[g_{20} r_{1}(0)+\bar{g}_{02} r_{2}(0)\right] \cdot f_{0} \\
& H_{11}(0)=\frac{\tau^{*}}{4}\left(\begin{array}{c}
-e^{-\rho \tau^{*}}\left(\beta_{1}+\beta_{2} \gamma_{5}\right)(\sigma+\bar{\sigma})-2 \beta_{2} P^{*} e^{-\rho \tau^{*}} \gamma_{6} \sigma \bar{\sigma}+\alpha \sigma \bar{\sigma}\left(e^{-i \omega \tau^{*}}+e^{i \omega \tau^{*}}\right) \\
e^{-\rho \tau^{*}}\left(\beta_{1}+\beta_{2} \gamma_{5}\right)(\sigma+\bar{\sigma})+2 \beta_{2} P^{*} e^{-\rho \tau^{*}} \gamma_{6} \sigma \bar{\sigma}-\alpha \sigma \bar{\sigma}\left(e^{-i \omega \tau^{*}}+e^{i \omega \tau^{*}}\right)
\end{array}\right) \\
& -\frac{1}{2}\left[g_{11} r_{1}(0)+\bar{g}_{11} r_{2}(0)\right] \cdot f_{0}
\end{aligned}
$$
that

According to the definition of $A_{\tau^{*}}$, and from (70), we get

$$
\begin{aligned}
\dot{W}_{20}(\theta)= & 2 i \omega_{0} \tau^{*} W_{20}(\theta)+\frac{1}{2}\left[g_{20} r_{1}(\theta)+\bar{g}_{02} r_{2}(\theta)\right] \\
& \cdot f_{0},-1 \leq \theta<0 .
\end{aligned}
$$

It is noted that $r_{1}(\theta)=r_{1}(0) e^{i \omega_{0} \tau^{*}}, \quad-1 \leq \theta \leq 0$.

$$
W_{20}(\theta)=\frac{i}{2}\left[\frac{g_{20}}{\omega_{0} \tau^{*}} r_{1}(\theta)+\frac{\bar{g}_{02}}{3 \omega_{0} \tau^{*}} r_{2}(\theta)\right] \cdot f_{0}+E e^{2 i \omega_{0} \tau^{*} \theta} \text {, }
$$

$$
E=W_{20}(0)-\frac{i}{2}\left[\frac{g_{20}}{\omega_{0} \tau^{*}} r_{1}(0)+\frac{\bar{g}_{02}}{3 \omega_{0} \tau^{*}} r_{2}(0)\right] \cdot f_{0} .
$$
Consequently,

$$
\begin{aligned}
2 i \omega_{0} \tau^{*} & {\left[\frac{i g_{20}}{2 \omega_{0} \tau^{*}} r_{1}(0) \cdot f_{0}+\frac{i \bar{g}_{02}}{6 \omega_{0} \tau^{*}} r_{2}(0) \cdot f_{0}+E\right]-L\left(\tau^{*}\right)\left[\frac{i g_{20}}{2 \omega_{0} \tau^{*}} r_{1}(\theta) \cdot f_{0}+\frac{i \bar{g}_{02}}{6 \omega_{0} \tau^{*}} r_{2}(\theta) \cdot f_{0}+E e^{2 i \omega_{0} \tau^{*} \theta}\right] } \\
\quad= & \frac{\tau^{*}}{2}\left(\begin{array}{c}
-e^{-\rho \tau^{*}}\left(\beta_{1}+\beta_{2} \gamma_{5}\right)-\beta_{2} P^{*} e^{-\rho \tau^{*}} \gamma_{6} \sigma^{2}+\alpha \sigma^{2} e^{-i \omega \tau^{*}} \\
e^{-\rho \tau^{*}}\left(\beta_{1}+\beta_{2} \gamma_{5}\right)+\beta_{2} P^{*} e^{-\rho \tau^{*}} \gamma_{6} \sigma^{2}-\alpha \sigma^{2} e^{-i \omega \tau^{*}}
\end{array}\right)-\frac{1}{2}\left[g_{20} r_{1}(0)+\bar{g}_{02} r_{2}(0)\right] \cdot f_{0} .
\end{aligned}
$$

Notice that

$$
\left\{\begin{array}{l}
L\left(\tau^{*}\right)\left[r_{1}(\theta) \cdot f_{0}\right]=i \omega_{0} \tau^{*} r_{1}(0) \cdot f_{0}, \\
L\left(\tau^{*}\right)\left[r_{2}(\theta) \cdot f_{0}\right]=-i \omega_{0} \tau^{*} r_{2}(0) \cdot f_{0}
\end{array}\right.
$$

Thus, we get

$$
\begin{aligned}
& 2 i \omega_{0} \tau^{*} E-\tau^{*} D \Delta E-L\left(\tau^{*}\right)\left(E e^{2 i \omega_{0} \tau^{*} \theta}\right) \\
& =\frac{\tau^{*}}{2}\left(\begin{array}{c}
-e^{-\rho \tau^{*}}\left(\beta_{1}+\beta_{2} \gamma_{5}\right)-\beta_{2} P^{*} e^{-\rho \tau^{*}} \gamma_{6} \sigma^{2}+\alpha \sigma^{2} e^{-i \omega \tau^{*}} \\
e^{-\rho \tau^{*}}\left(\beta_{1}+\beta_{2} \gamma_{5}\right)+\beta_{2} P^{*} e^{-\rho \tau^{*}} \gamma_{6} \sigma^{2}-\alpha \sigma^{2} e^{-i \omega \tau^{*}}
\end{array}\right) .
\end{aligned}
$$

From the above equation, it is easily obtained that

$$
E=\frac{1}{2}\left(\begin{array}{cc}
2 i \omega_{0}-\gamma_{1} & -\gamma_{3}+\beta_{2} P^{*} e^{-\rho \tau} \gamma_{2}-\alpha A^{*} e^{-2 i \omega_{0} \tau^{*}} \\
-\gamma_{4} & 2 i \omega_{0}-\beta_{2} P^{*} e^{-\rho \tau} \gamma_{2}+\alpha A^{*} e^{-2 i \omega_{0} \tau^{*}}
\end{array}\right)^{-1}
$$

$$
\times\left(\begin{array}{c}
-e^{-\rho \tau^{*}}\left(\beta_{1}+\beta_{2} \gamma_{5}\right)-\beta_{2} P^{*} e^{-\rho \tau^{*}} \gamma_{6} \sigma^{2}+\alpha \sigma^{2} e^{-i \omega \tau^{*}} \\
e^{-\rho \tau^{*}}\left(\beta_{1}+\beta_{2} \gamma_{5}\right)+\beta_{2} P^{*} e^{-\rho \tau^{*}} \gamma_{6} \sigma^{2}-\alpha \sigma^{2} e^{-i \omega \tau^{*}}
\end{array}\right) .
$$

Similarly, we obtain 


$$
\begin{aligned}
& \dot{W}_{11}(\theta)=\frac{1}{2}\left[g_{11} r_{1}(\theta)+\bar{g}_{11} r_{2}(\theta)\right] \cdot f_{0}, \quad-1 \leq \theta<0, \\
& W_{11}(\theta)=\frac{i}{2 \omega_{0} \tau^{*}}\left[-g_{11} r_{1}(\theta)+\bar{g}_{11} r_{2}(\theta)\right] \cdot f_{0}+F
\end{aligned}
$$

In the similarly way, we get

$$
\begin{aligned}
F= & \frac{1}{4}\left(\begin{array}{cc}
-\gamma_{1} & -\gamma_{3}+\beta_{2} P^{*} e^{-\rho \tau} \gamma_{2} \\
-\gamma_{4} & -\beta_{2} P^{*} e^{-\rho \tau} \gamma_{2}
\end{array}\right)^{-1} \\
& \times\left(\begin{array}{c}
-e^{-\rho \tau^{*}}\left(\beta_{1}+\beta_{2} \gamma_{5}\right)(\sigma+\bar{\sigma})-2 \beta_{2} P^{*} e^{-\rho \tau^{*}} \gamma_{6} \sigma \bar{\sigma}+\alpha \sigma \bar{\sigma}\left(e^{-i \omega \tau^{*}}+e^{i \omega \tau^{*}}\right) \\
e^{-\rho \tau^{*}}\left(\beta_{1}+\beta_{2} \gamma_{5}\right)(\sigma+\bar{\sigma})+2 \beta_{2} P^{*} e^{-\rho \tau^{*}} \gamma_{6} \sigma \bar{\sigma}-\alpha \sigma \bar{\sigma}\left(e^{-i \omega \tau^{*}}+e^{i \omega \tau^{*}}\right)
\end{array}\right) .
\end{aligned}
$$

As a result, $g_{21}$ can be obtained explicitly, and we have the following result.

Theorem 3. System (4) has the following Poincare normal form

$$
\dot{\xi}=i \omega_{0} \tau^{*} \xi+c_{1}(0) \xi|\xi|^{2}+o\left(|\xi|^{5}\right)
$$

where

$$
c_{1}(0)=\frac{i}{2 \omega_{0} \tau^{*}}\left[g_{20} g_{11}-2\left|g_{11}\right|^{2}-\frac{\left|g_{02}\right|^{2}}{3}\right]+\frac{g_{21}}{2} .
$$

Hence, we can compute the following results:

$$
\begin{aligned}
& \sigma_{2}=-\frac{\operatorname{Re}\left(c_{1}(0)\right)}{\operatorname{Re}\left(\lambda^{\prime}\left(\tau^{*}\right)\right)} \\
& \beta_{2}=2 \operatorname{Re}\left(c_{1}(0)\right) \\
& T_{2}=-\frac{\operatorname{Im}\left(c_{1}(0)\right)+\sigma_{2} \operatorname{Im}\left(\lambda^{\prime}\left(\tau^{*}\right)\right)}{\omega_{0} \tau^{*}}
\end{aligned}
$$

which determine the properties of bifurcating periodic solutions at the critical values $\tau^{*}$; i.e., $\sigma_{2}$ determines the directions of the Hopf bifurcation: if $\sigma_{2}>0\left(\sigma_{2}<0\right)$, then the Hopf bifurcation is supercritical (subcritical) and the bifurcating periodic solutions exist for $\tau>\tau^{*} ; \beta_{2}$ determines the stability of the bifurcating periodic solutions: the bifurcating periodic solutions on the center manifold are stable (unstable) if $\beta_{2}<0\left(\beta_{2}>0\right)$; and $T_{2}$ determines the period of the bifurcating periodic solutions: the periodic increase (decrease) occurs if $T_{2}>0\left(T_{2}<0\right)$.

\section{Numerical Simulation}

In this section, we present numerical simulations of some examples to illustrate our theoretical results.

5.1. Hopf Bifurcations Induced by Delay. Consider system (4) with the following parameters: $r=0.8, \alpha=0.3, \beta_{1}=0.3$, $\beta_{2}=0.2, \delta=0.04, m=10$, and $\rho=0.4$. We plot the graph of $P^{*}$ and $A^{*}$ and that of $S_{j}(\tau)$ versus $\tau$ in the interval $[0,10]$ in Figures 1 and 2, respectively.

From Figure 1, we find that, with increasing delay $\tau, P^{*}$ gradually increases and $A^{*}$ gradually decrease, and when $\tau \approx 11.5$, the positive equilibrium disappears. In fact, the positive equilibrium and the boundary equilibrium $E_{0}$ are inconsistent at this time. This outcome occurs because when the evaluation period of a new product becomes very long, the individuals do not become adopters since they do not adopt the product.

Apparently, no zero point exits for $S_{1}(\tau)$, and for $S_{0}(\tau)$, there are two zeros: the first at $\tau_{1}=1.0879$ and the other at $\tau_{2}=6.6616$. These indicate that, at the point $\tau \in\left[0, \tau_{1}\right)$, the positive equilibrium of system (4) is stable (see Figure 3 ), and when $\tau \in\left(\tau_{1}, \tau_{2}\right)$, the positive equilibrium of system (4) is unstable (see Figure 4). As $\tau$ increases through the critical value $\tau_{2}=6.6616$, the stability switch occurs again, and the positive equilibrium of system (4) becomes stable, as shown in Figure 5.

When $\tau$ passes through the zero point $\tau_{1}=1.0879$, the positive equilibrium becomes unstable, and a Hopf bifurcation occurs, i.e., a family of periodic solutions bifurcate from the positive equilibrium, as shown in Figure 4 . We can compute $\mu_{2}=-\operatorname{Re}\left(c_{1}(0)\right) / \operatorname{Re}\left(\lambda^{\prime}\left(\tau^{*}\right)\right)=$ $22243672.8653>0$ and $\beta_{2}=2 \operatorname{Re}\left(c_{1}(0)\right)=-109.2516<0$. Therefore, the orbitally asymptotically bifurcated periodic solutions of the system are stable (4) when $\tau_{1}=1.1929$ in the whole phase space, and the Hopf bifurcation is supercritical for $\mu_{2}>0$. As $\tau$ increases further and reaches the critical value $\tau_{2}=6.6616$, it can be computed that $\beta_{2}=0.3038$. Hence, the limit cycle becomes unstable based on the discussion in Section 3, and the positive equilibrium recovers it stability, as shown in Figure 5. In summary, with the increase in $\tau$, the positive equilibrium of the system will finally reach stability by two Hopf bifurcations. However, if the delay $\tau$ increases further, then the positive equilibrium will disappear, and all solutions of system (4) will reach equilibrium $E_{0}$, as shown in Figure 6. 


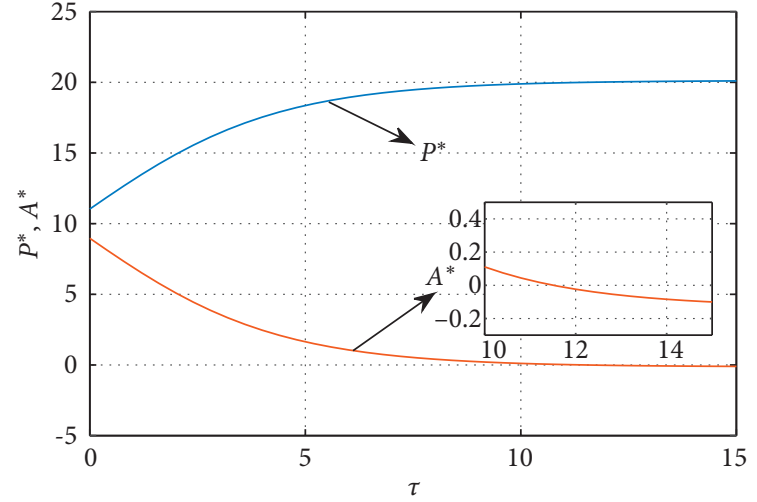

Figure 1: Graph of $P^{*}$ and $A^{*}$ versus $\tau$ for system (4).

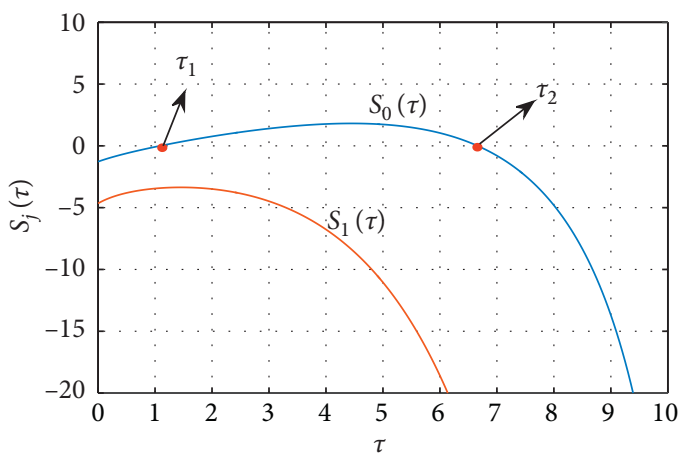

Figure 2: Graph of $S_{j}(\tau)$ versus $\tau$ for system (4).

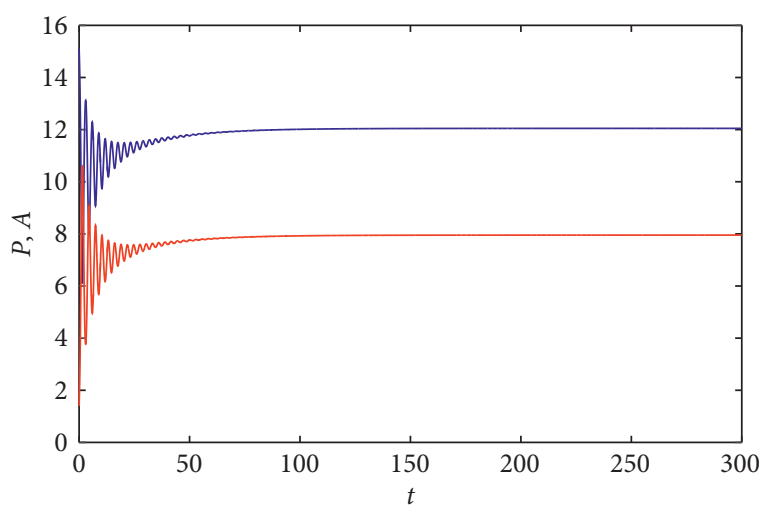

FIgURE 3: The equilibrium $E^{*}$ of system (4) is stable with $\tau=0.9$.

5.2. Effect of Parameter $\rho$. We now investigate the effect of the parameter $\rho$. Therefore, let the parameters be the same as in Section 5.1, except $\rho=0$, which means that the system parameters are constants independent of time delay. The graph of $S_{j}(\tau)$ versus $\tau$ is plotted in Figure 7. It is shown that the positive equilibrium is stable with $\tau \in\left[0, \tau_{1}\right)$.

However, with each crossing of every critical delay $\tau_{j}(j=1,2, \ldots$,$) , the number of eigenvalues with positive$

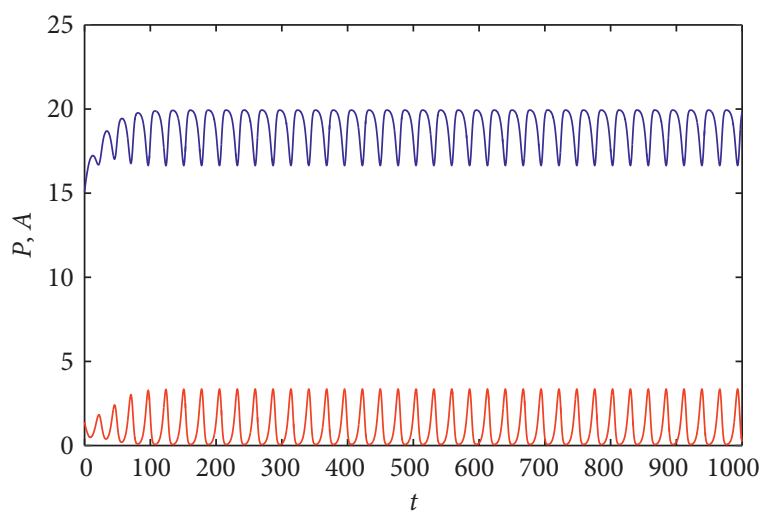

Figure 4: The equilibrium $E^{*}$ of system (4) is unstable with $\tau=6$.

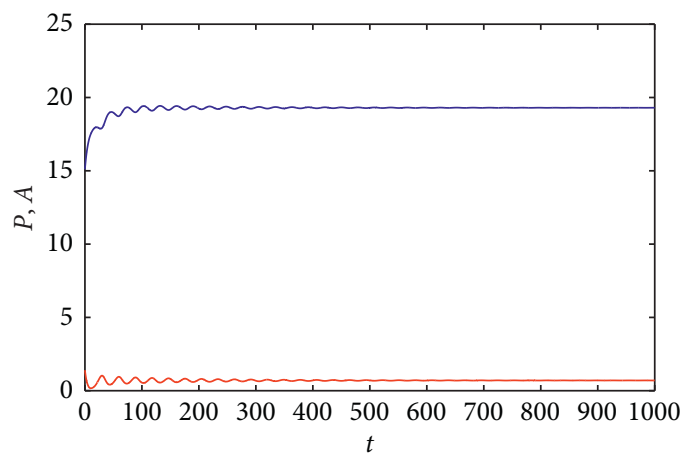

FIgURE 5: The equilibrium $E^{*}$ of system (4) is stable with $\tau=8.4$.

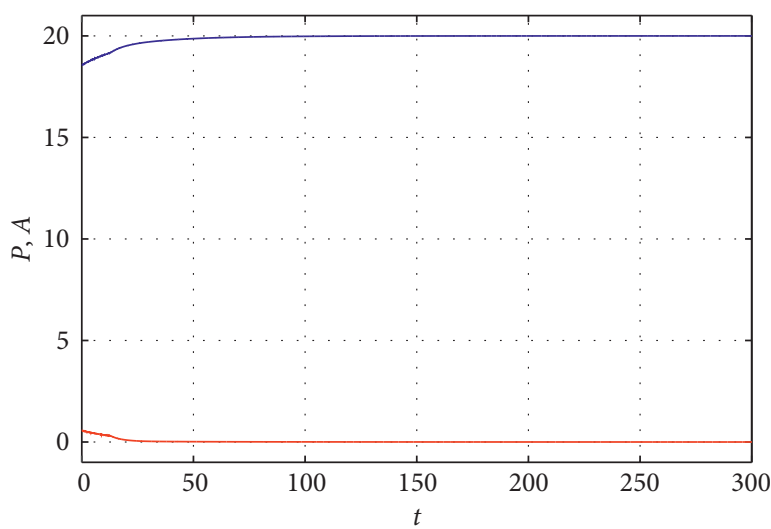

FIGURE 6: All solutions of system (4) converge to the equilibrium $E_{0}$ with $\tau=13$.

real parts can be increased by 2 , and the equilibrium becomes unstable. This is quite different from the analysis in Figure 2. In this analysis, with the increase in $\tau$, a limited number of stable switches can occur, and the system can be stabilized with a moderately large delay.

Figure 8 shows the graph of $S_{j}(\tau)$ versus $\tau$ for different $\rho$. Clearly, the larger the value of $\rho$ is, the wider the interval of the time delay $\tau$ is under system stability. 


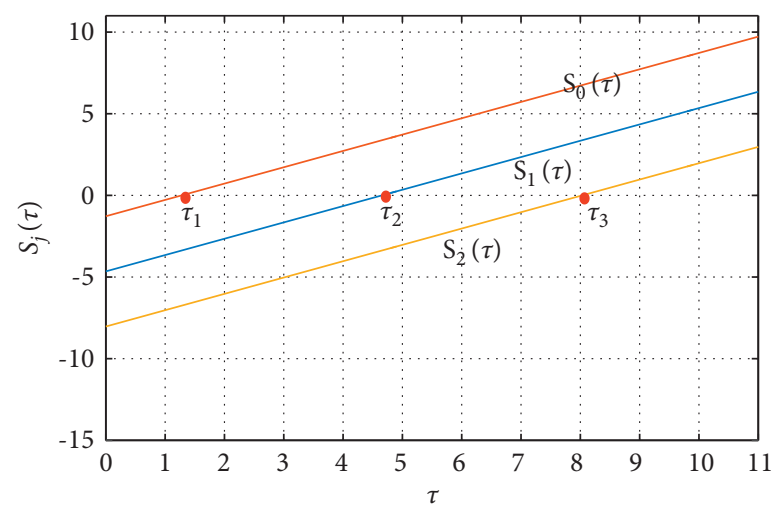

Figure 7: Graph of $S_{j}(\tau)$ versus $\tau$ for the neuron model (4) with delay-independent parameters.

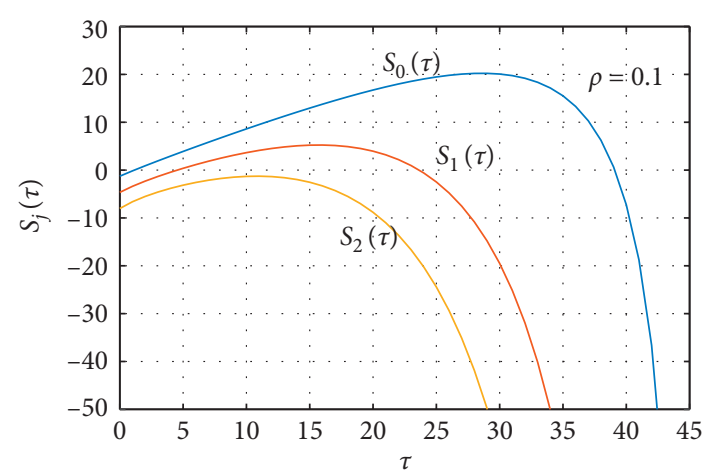

(a)

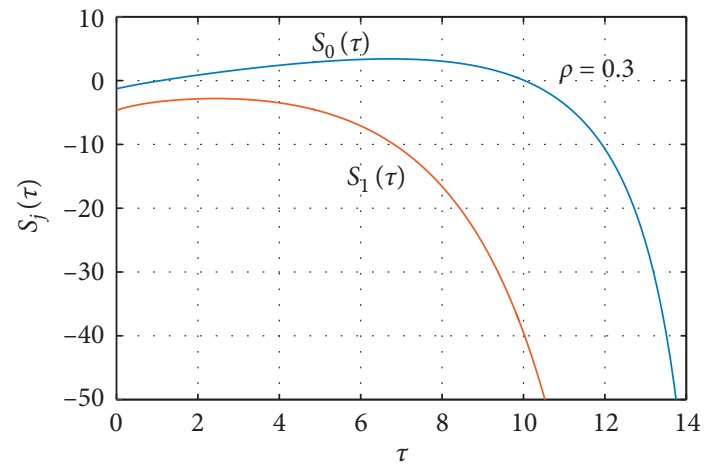

(c)

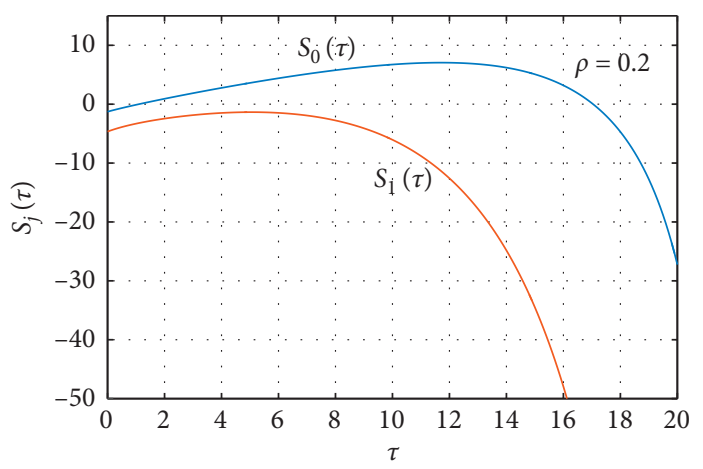

(b)

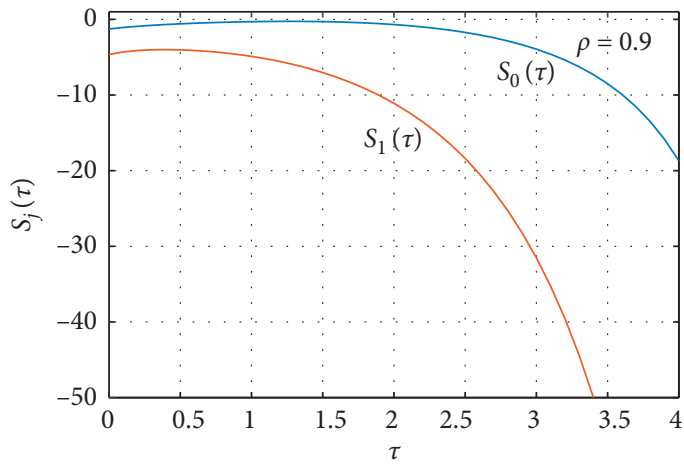

(d)

FIGURE 8: The effect of parameter $\rho$ on the stability of system.

\section{Conclusions}

In this paper, the stability switches and Hopf bifurcation of a delayed nonlinear mathematical model are analyzed in detail. The model is designed with a stage structure used to simulate the stages of the process of adopting a new product. Moreover, the normal and the center manifold theory are used to investigate the stability and the direction of the bifurcating periodic solutions. The results of the numerical simulations show that, in the range of zero to infinity for the delay, there may exist a number of stability switches for the model with delay-dependent parameters. Moreover, the system could also be stabilized by a moderately large delay. This characteristic of the models is quite different from that of systems without delay-dependent parameters.

\section{Data Availability}

The data used to support the findings of this study are included within the article.

\section{Conflicts of Interest}

The authors declare that they have no conflicts of interest. 


\section{Acknowledgments}

The work was supported by the National Science Foundation of China under Grant no. 71672154.

\section{References}

[1] L. A. Fourt and J. W. Woodlock, "Early prediction of market success for new grocery products," Journal of Marketing, vol. 25, no. 2, pp. 31-38, 1960.

[2] F. Bass, A New Product Growth Model for Consumer Durables, Springer Berlin Heidelberg, Berlin, Germany, 1969.

[3] D. Horsky and L. S. Simon, "Advertising and the diffusion of new products," Marketing Science, vol. 2, no. 1, pp. 1-17, 1983.

[4] D. Horsky, "A difffusion model incorporating product benefits, price, income and information," Marketing Science, vol. 2, 2010.

[5] D. C. Jain and R. C. Rao, "Effect of price on the demand for durables: modeling, estimation, and findings," Journal of Business \& Economic Statistics, vol. 8, no. 2, pp. 163-170, 1990.

[6] S. Kalish, "A new product adoption model with price, advertising, and uncertainty," Management Science, vol. 31, no. 12, pp. 1569-1585, 1985.

[7] B. Robinson and C. Lakhani, "Dynamic price models for newproduct planning," Management Science, vol. 21, no. 10, pp. 1113-1122, 1975.

[8] D. Satoh, "A discrete bass model and its parameter estimation," Journal of the Operations Research Society of Japan, vol. 44 , no. 1, pp. 1-18, 2001.

[9] Z. He and J. Kan, Footprint of New Product in Mobile Market Using Diffusion Models, Springer International Publishing, Berlin, Germany, 2014.

[10] S.-C. Niu, "A piecewise-diffusion model of new-product demands," Operations Research, vol. 54, no. 4, pp. 678-695, 2006.

[11] S. Kalish and G. L. Lilien, "A market entry timing model for new technologies," Management Science, vol. 32, no. 2, pp. 194-205, 1986.

[12] M. L. Bertotti, J. Brunner, and G. Modanese, "Innovation diffusion equations on correlated scale-free networks," Physics Letters A, vol. 380, no. 33, pp. 2475-2479, 2016.

[13] J. Dhar, M. Tyagi, and P. Sinha, "An innovation diffusion model for the survival of a product in a competitive market: basic influence numbers," Japanese Journal of Applied Physics, vol. 89, no. 4, pp. 6623-6624, 2013.

[14] J. Dhar, M. Tyagi, and P. Sinha, "Dynamical behavior in an innovation diffusion marketing model with thinker class of population," International Journal of Business Management Economics and Information Technology, vol. 1, no. 1, pp. 7984,2010

[15] Y. Yu, W. Wang, and Y. Zhang, "An innovation diffusion model for three competitive products," Computers \& Mathematics with Applications, vol. 46, no. 10-11, pp. 1473-1481, 2003.

[16] V. Fanelli and L. Maddalena, "A time delay model for the diffusion of a new technology," Nonlinear Analysis Real World Applications, vol. 13, no. 2, pp. 643-649, 2013.

[17] L. V. Ballestra, L. Guerrini, and G. Pacelli, "Stability switches and bifurcation analysis of a time delay model for the diffusion of a new technology," International Journal of Bifurcation and Chaos, vol. 24, no. 9, Article ID 1450113, 2014.

[18] J. Dhar, M. Tyagi, and P. Sinha, "The impact of media on a new product innovation diffusion: a mathematical model,"
Boletim da Sociedade Paranaense de Matemática, vol. 33, no. 1, pp. 171-182, 2015.

[19] E. Beretta and Y. Kuang, "Geometric stability switch criteria in delay differential systems with delay dependent parameters," SIAM Journal on Mathematical Analysis, vol. 33, no. 5, pp. 1144-1165, 2002.

[20] J. Wu, Theory and Applications of Partial Functional Differential Equations, Applied Mathematical Sciences. Springer, New York, NY, USA, 1996. 